\title{
Credit Risk and the Instability of the Financial System: an Ensemble Approach
}

\author{
Thilo A. Schmitt* Desislava Chetalova, Rudi Schäfer, and Thomas Guhr \\ Fakultät für Physik, Universität Duisburg-Essen, Duisburg, Germany
}

(Dated: August 22, 2018)

\begin{abstract}
The instability of the financial system as experienced in recent years and in previous periods is often linked to credit defaults, i.e., to the failure of obligors to make promised payments. Given the large number of credit contracts, this problem is amenable to be treated with approaches developed in statistical physics. We introduce the idea of ensemble averaging and thereby uncover generic features of credit risk. We then show that the often advertised concept of diversification, i.e., reducing the risk by distributing it, is deeply flawed when it comes to credit risk. The risk of extreme losses remains due to the ever present correlations, implying a substantial and persistent intrinsic danger to the financial system.
\end{abstract}

\section{INTRODUCTION}

The past years demonstrated the devastating consequences when financial markets collapse. The instability of the financial system is closely connected to that of banks and related institutions which, in turn, is directly coupled to the losses when the obligors, i.e., the companies or individuals that borrowed money, default and are unable to fully repay. In the recession following a market break down, a higher than usual number of defaults occur [1], severely worsening the situation. The crisis of 2007-2009 was triggered by false assessment of the risk involved with subprime mortgage credits [2]. The ensuing bankruptcy of Lehman Brothers 3 then released an avalanche effecting the world economy as a whole. Economists who saw the problems piling up have pointed out the importance of improved credit risk estimation [48. However, a quantitative study satisfying the standards common in physics is missing. Here, we want to close this gap by transferring a standard tool from statistical physics, namely ensemble average, to credit risk estimation.

The problem can be traced back to the peculiar shape of a loss distribution $p(L)$ for a portfolio consisting of a large number of credit contracts. It is the probability density function (pdf) of the dimensionless loss $L$ relative to the total exposure, i.e., to the entire amount of money given out in the credits. Typically, an empirical loss distribution looks as shown in Fig. 1. The asymmetry and the heavy tail on the right hand side are striking. They are caused by the specific properties of the credit contracts: The highest possible gain for the bank issuing the credits is only due to interest and risk compensation, depending on the creditworthiness. It occurs only if not a single credit defaults. On the other hand, the largest possible loss results from a complete loss of the lent money. The danger lies in the heavy tail, which describes the probability for large losses that exceed the possible gains by far. Individual, large defaults such as Enron or Lehmann Brothers as well as simultaneous de-

*Electronic address: thilo.schmitt@uni-due.de

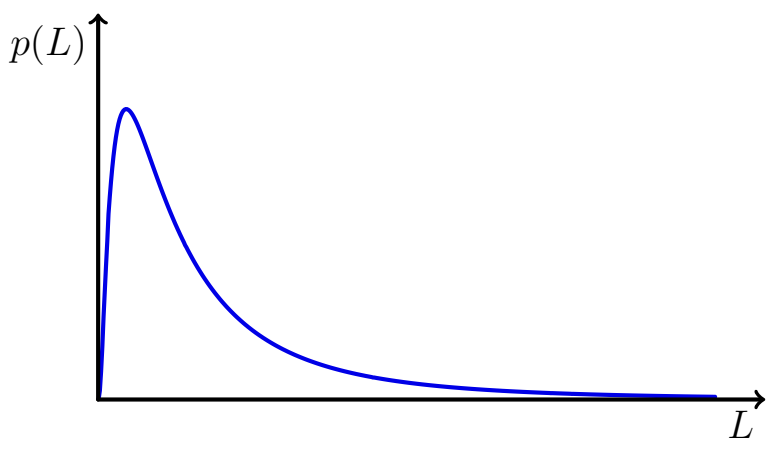

FIG. 1: Schematic drawing of a typical loss distribution $p(L)$ versus the relative loss $L$.

faults of many small obligors as in the subprime mortgage crisis are the events making this tail so heavy.

Thus, the issue of instability can be reformulated as the question whether or not it is possible to get rid of this heavy tail. Financial institutions often claim that this can be achieved by simply enlarging the number of obligors and credit contracts in the portfolio. The resulting diversification is then believed to reduce the risk for the bank. This view has been severely criticized, both with qualitative reasoning [9] and quantitative studies addressing this important issue in the economics literature, see e.g., 10 13. Intuitively, it is not difficult to understand why the concept of diversification is highly questionable. If the obligors are correlated by some mutual dependencies, the default events will appear clustered. Only in the economically unrealistic case of zero correlations, diversification can work. Our goal is to identify generic features of credit risk using a standard approach from statistical physics: an ensemble approach for correlations. As an application, we then show that diversification is bound to fail. We even derive an exact limiting loss distribution. 


\section{MERTON MODEL}

We use a "microscopic" model, referred to as "structural" in economics, put forward by Merton 14. While "reduced-form" models, see e.g., [6, 11, 15], only provide an abstract description of default events, the structural model traces defaults and losses back to stochastic processes describing the economic state of each individual obligor. Suppose $K$ obligors hold credit contracts which bind them to pay back the amount of money $F_{k}, k=1, \ldots, K$, referred to as face value, at the maturity time $T$. The stochastic variable $V_{k}(t)$ is the economic state, i.e., the value of the $k$-th company, which can be retrieved after a bankruptcy and paid to the creditors. As Fig. 2 shows, the stochastic process now leads to a

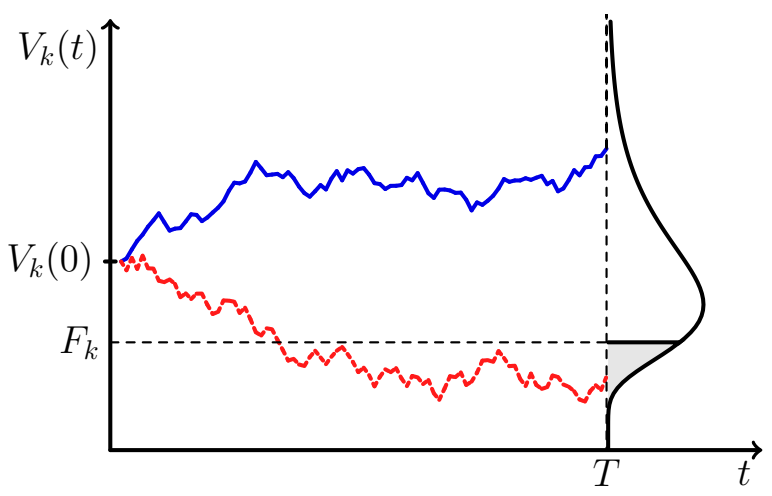

FIG. 2: Sketch of the Merton model. Default occurs if the economic state $V_{k}$ falls below the face value $F_{k}$ at maturity time $T$.

distribution of outcomes $V_{k}(T)$ at maturity. In the cases $V_{k}(T) \geq F_{k}$, the obligor is able to make the promised payment, the cases $V_{k}(T)<F_{k}$ are default events. This does not necessarily mean that the whole face value $F_{k}$ is lost. Rather, the normalized dimensionless loss of contract $k$ is

$$
L_{k}=\frac{F_{k}-V_{k}(T)}{F_{k}} \Theta\left(F_{k}-V_{k}(T)\right) .
$$

The Heaviside function ensures that the loss is strictly positive, because only the default events are to be taken into account. The entire credit portfolio comprises the individual losses of all $K$ contracts. The corresponding portfolio loss is the sum of the individual losses $L_{k}$ weighted by their fraction $f_{k}$ in the portfolio

$$
L=\sum_{k=1}^{K} f_{k} L_{k} \quad, \quad f_{k}=\frac{F_{k}}{\sum_{i=1}^{K} F_{i}} .
$$

The distribution of the portfolio loss is then given by

$$
p(L)=\int d[V] g(V \mid \Sigma) \delta\left(L-\sum_{k=1}^{K} f_{k} L_{k}\right)
$$

where $g(V \mid \Sigma)$ is the multivariate distribution of the economic states at maturity, $V=\left(V_{1}(T), \ldots, V_{K}(T)\right)$. Importantly, it depends on the $K \times K$ covariance matrix $\Sigma$ measuring the above mentioned mutual dependencies between the obligors. The innocent-looking integral (3) is a highly non-trivial object, first, because the $L_{k}$ contain, according to Eq. (1), a Heaviside function and, second, because the multivariate distribution $g(V \mid \Sigma)$ of the economic states at maturity is unknown. It was Merton's seminal idea to estimate the stochastic processes $V_{k}(t)$ by the stock prices $S_{k}(t)$, provided all $K$ obligors are companies listed on the stock market. We assume this from now on. In this Merton model it has been shown by numerical simulations [16, 17 that the heavy tail of the loss distribution remains in the presence of even weak correlations. In a less realistic setting, referred to as first passage model with constant recovery and correlated defaults, this had already been found in the economics literature [10, 18].

\section{ENSEMBLE AVERAGE}

Although the empirical stock market data give us, in principle, access to $g(V \mid \Sigma)$, the formidable complexity of the stock market dynamics keeps us still far from achieving a generic understanding beyond the numerical case studies of Ref. [16]. The crucial problem is the nonstationarity which is commonly studied for the returns, i.e., for the dimensionless differences of the stock prices $S_{k}(t)$

$$
r_{k}(t)=\frac{S_{k}(t+\Delta t)-S_{k}(t)}{S_{k}(t)}
$$

where $\Delta t$ is referred to as return horizon. Neither the standard deviations or volatilities $\sigma_{k}$ of the individual returns $r_{k}(t)$, nor the correlations $C_{k l}$ of any pair $\left(r_{k}(t), r_{l}(t)\right)$ are constant in time. We recall the relation $\Sigma=\sigma C \sigma$ with $\sigma=\operatorname{diag}\left(\sigma_{1}, \ldots, \sigma_{K}\right)$ between covariance and correlation matrix. To handle this non-stationarity new concepts are called for. Here, we transfer the idea of ensemble averaging from statistical physics. To the best of our knowledge, this whole approach is new in the economics literature. According to Eq. (3), we need $g(V \mid \Sigma)$ which is obtained from the more easily accessible distribution $g(r \mid \Sigma)$ depending on the $K$ component vector of the returns (4). Recently, we constructed this distribution and confirmed its validity by a careful data analysis [19. We showed that $g\left(r \mid \Sigma_{s}\right)$ is a multivariate Gaussian,

$$
g\left(r \mid \Sigma_{s}\right)=\frac{1}{\sqrt{\operatorname{det}\left(2 \pi \Sigma_{s}\right)}} \exp \left(-\frac{1}{2} r^{\dagger} \Sigma_{s}^{-1} r\right)
$$

if the data are sampled in a data interval short enough that $\Sigma_{s}$ is constant 20. Importantly, we are interested in maturity times $T$ of at least a month or so. As the 
maturity time sets the return horizon, $T=\Delta t$, the wellknown heavy tails of the individual return distributions are not so pronounced yet. Moreover, as we consider the multivariate distribution of all returns, the heavy tails of the individual return distributions are further suppressed. However, we are interested not in short, but in larger data intervals (not to be confused with the return horizon) where sizable non-stationarity is present. We take it into account by averaging over the correlation matrices. We use the Wishart distribution [21 of the correlation matrices $W W^{\dagger}$,

$$
w(W \mid C, N)=\frac{\sqrt{N}^{K N}}{\sqrt{\operatorname{det}(2 \pi C)}^{N}} \exp \left(-\frac{N}{2} \operatorname{tr} W^{\dagger} C^{-1} W\right),
$$

which defines an ensemble of correlation matrices $W W^{\dagger}$ that fluctuate around the mean correlation matrix $C$, calculated for the entire data interval. The model matrices $W$ have dimension $K \times N$, where $N$ formally corresponds to the length of the model time series. It is a free parameter to be determined from the data later on. It governs the variance of the distribution (6) and thus the strength of the fluctuations around $C$. We break the entire data interval into many short ones for which the observation (5) is justified and we put $\Sigma_{s}=\sigma W W^{\dagger} \sigma$. The average

$$
\langle g\rangle(r \mid \Sigma, N)=\int d[W] w(W \mid C, N) g\left(r \mid \sigma W W^{\dagger} \sigma\right)
$$

then accounts for the non-stationarity. The result only depends on $\Sigma=\sigma C \sigma$, calculated over the entire data interval, it reads

$$
\begin{aligned}
\langle g\rangle(r \mid \Sigma, N) & =\frac{\sqrt{N}^{K}}{\sqrt{2}^{N-2} \Gamma(N / 2) \sqrt{\operatorname{det}(2 \pi \Sigma)}} \\
& \frac{\mathcal{K}_{(K-N) / 2}\left(\sqrt{N r^{\dagger} \Sigma^{-1} r}\right)}{{\sqrt{N r^{\dagger} \Sigma^{-1} r}}^{(K-N) / 2}}
\end{aligned}
$$

with the Bessel function $\mathcal{K}$ of the second kind of order $(K-N) / 2$. We demonstrated the validity of this result by obtaining $\Sigma$ directly from the data and by fitting $N$ [19, 20. Here, however, it is advantageous to make the additional approximation that all off-diagonal correlation matrix elements are equal, i.e., $C_{k l}=c, k \neq l$, hence

$$
C=(1-c) \mathbb{1}_{K}+c e e^{\dagger}
$$

where $\mathbb{1}_{K}$ is the $K \times K$ unit matrix and $e$ is a $K$ component vector with unity in all entries. By averaging all off-diagonal matrix elements of $C$ measured in the whole data interval, we find $c=0.26$ for monthly and $c=0.28$ for yearly returns. The data set consists of 306 stocks from the S\&P 500-index in the time interval from 1992 to 2012 22]. To test our result (8) with the approximation $(9)$, we rotate the returns into the eigenbasis of $\Sigma$ and scale with the eigenvalues. Integrating out all but one rescaled return, denoted $\tilde{r}$, we have

$\langle g\rangle(\tilde{r} \mid N)=\frac{\sqrt{2}^{1-N} \sqrt{N}}{\sqrt{\pi} \Gamma(N / 2)}{\sqrt{N \tilde{r}^{2}}}^{(N-1) / 2} \mathcal{K}_{(N-1) / 2}\left(\sqrt{N \tilde{r}^{2}}\right)$.

Figure 3 shows the fit to the data which determines the value of $N$. The fit for return horizons $\Delta t$ of a month

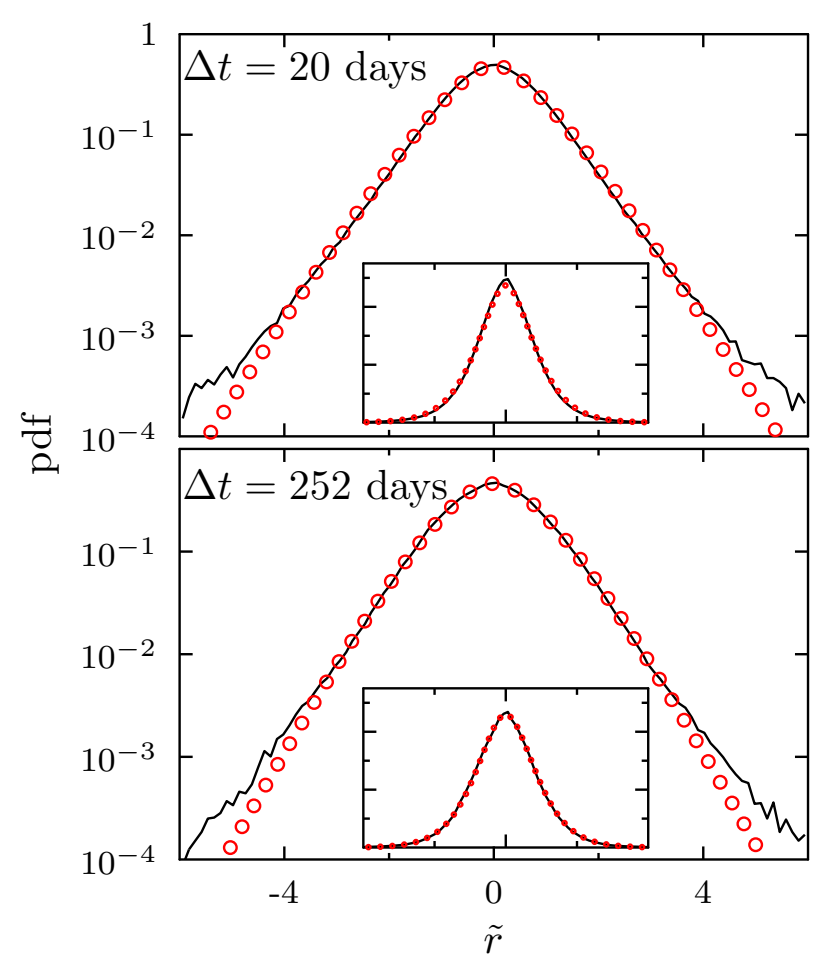

FIG. 3: Aggregated distribution of the rotated and rescaled returns $\tilde{r}$ for monthly returns $\Delta t=20$ trading days (top) and yearly returns $\Delta t=252$ trading days (bottom) for the model covariance matrix. The insets show the linear-linear plot.

or a year - which later on carries over to the maturity times $T$ - is very good with $N=4.2$ and $N=6.0$, respectively. The tails are heavy, deviations only appear beyond the third decade.

\section{AVERAGED LOSS DISTRIBUTION}

The merit of the above construction is the drastic reduction in the number of degrees of freedom. The return distribution of the highly complex, non-stationary market is now fully and quantitatively characterized by the two parameters $c$ and $N$ measuring the mean and the variance of the fluctuations. This identification of generic features out of a very large number of quantities is reminiscent of statistical mechanics where a few macroscopic variables characterize a large system that is microscopically described by a huge number of variables. Hence, 
we are now able to also uncover generic features of credit risk. Here, we greatly expand our previous work 23] to the more realistic case of a non-zero average correlation level and, in addition, we give an empirical verification of our approach. According to Eq. (3), the averaged loss distribution reads

$$
\langle p\rangle(L)=\int d[V]\langle g\rangle(V \mid \Sigma, N) \delta\left(L-\sum_{k=1}^{K} f_{k} L_{k}\right) .
$$

We infer the price distribution $\langle g\rangle(V \mid \Sigma, N)$ from our result (8) for the returns. The assumption that the stock prices $S_{k}(t)$ follow a Geometric Brownian Motion with drift and volatility constants $\mu_{k}$ and $\rho_{k}$, respectively, leads to a multivariate Gaussian of the form (5) for the returns. Hence, this is fully consistent with the above ensemble approach. Our data comparison thus strongly corroborates the usage of the Geometric Brownian Motion.

To make analytical progress, we write the return distribution (8) as a Fourier integral in the $K$ component vector $\omega$

$$
\begin{aligned}
\langle g\rangle(r \mid \Sigma, N)= & \frac{1}{2^{N / 2} \Gamma(N / 2)} \int_{0}^{\infty} d z z^{N / 2-1} \exp \left(-\frac{z}{2}\right) \\
& \int \frac{d[\omega]}{(2 \pi)^{K}} \exp \left(-\mathrm{i} \omega \cdot r-\frac{z}{2 N} \omega^{\dagger} \Sigma \omega\right) .
\end{aligned}
$$

We insert the approximation $(9)$ and linearize the square of the scalar product $\omega \cdot e$ in the exponent by another Fourier transform in $u$, say. The $\omega$ integral is then trivial and we find after straightforward steps

$$
\begin{aligned}
\langle g\rangle(r \mid c, N) & =\frac{1}{2^{N / 2} \Gamma(N / 2)} \frac{1}{\operatorname{det} \sigma} \int_{0}^{\infty} d z z^{N / 2-1} e^{-z / 2} \\
& \times \sqrt{\frac{N}{2 \pi z}} \sqrt{\frac{N}{2 \pi z(1-c)}} \int_{-\infty}^{\infty} d u \exp \left(-\frac{N}{2 z} u^{2}\right) \\
& \times \exp \left(-\sum_{k=1}^{K} \frac{N}{2 z(1-c) \sigma_{k}^{2}}\left(r_{k}+\sqrt{c} u \sigma_{k}\right)^{2}\right) .
\end{aligned}
$$

Following Merton but extending his idea to our ensemble approach, we now use the averaged return distribution to estimate the averaged distribution $\langle g\rangle(V \mid \Sigma, N)$ of the economic states $V_{k}(T)$ at maturity. According to Itō's Lemma 24] we set

$$
r_{k} \longrightarrow \ln \frac{V_{k}(T)}{V_{k 0}}-\left(\mu_{k}-\frac{\rho_{k}^{2}}{2}\right) T
$$

with $V_{k 0}=V_{k}(0)>0$. We notice $\sigma_{k}=\rho_{k} \sqrt{T}$. Inserting this into Eq. (11) yields the exact expression for the averaged loss distribution within our model.
To enforce simplicity, we consider a credit portfolio in which all face values are of the same order, implying that $f_{k} \approx 1 / K$. As the number of obligors $K$ is large, we may safely carry out a second order approximation in $1 / K$. Again, after tedious but straightforward steps, we eventually arrive at the double integral representation

$$
\begin{aligned}
\langle p\rangle(L \mid c, N)= & \frac{1}{\sqrt{2 \pi} 2^{N / 2} \Gamma(N / 2)} \int_{0}^{\infty} d z z^{N / 2-1} e^{-z / 2} \sqrt{\frac{N}{2 \pi}} \\
& \times \int_{-\infty}^{+\infty} d u \exp \left(-\frac{N}{2} u^{2}\right) \frac{1}{\sqrt{M_{2}(z, u)}} \\
& \times \exp \left(-\frac{\left(L-M_{1}(z, u)\right)^{2}}{2 M_{2}(z, u)}\right)
\end{aligned}
$$

for the average loss distribution. Here, we have

$$
\begin{aligned}
& M_{1}(z, u)=\sum_{k=1}^{K} f_{k} m_{1 k}(z, u) \\
& M_{2}(z, u)=\sum_{k=1}^{K} f_{k}^{2}\left(m_{2 k}(z, u)-m_{1 k}(z, u)^{2}\right)
\end{aligned}
$$

with the moments of order $j=1,2$ given by

$$
\begin{aligned}
m_{j k}(z, u)= & \frac{\sqrt{N}}{\rho_{k} \sqrt{2 \pi T(1-c)}} \int_{-\infty}^{\hat{F}_{k}} d \hat{V}_{k} \\
& \left(1-\frac{V_{k 0}}{F_{k}} \exp \left(\sqrt{z} \hat{V}_{k}+\left(\mu_{k}-\frac{\rho_{k}^{2}}{2}\right) T\right)\right)^{j} \\
& \times \exp \left(-\frac{\left(\hat{V}_{k}+\sqrt{c T} u \rho_{k}\right)^{2}}{2 T(1-c) \rho_{k}^{2} / N}\right)
\end{aligned}
$$

The upper bound of integration is $\hat{F}_{k}=\left(\ln \left(F_{k} / V_{k 0}\right)-\right.$ $\left.\left(\mu_{k}-\rho_{k}^{2} / 2\right) T\right) / \sqrt{z}$ and the change of variables leads to $\hat{V}_{k}=\left(\ln \left(V_{k}(T) / V_{k 0}\right)-\left(\mu_{k}-\rho_{k}^{2} / 2\right) T\right) / \sqrt{z}$. Since the integral can be expressed in terms of special functions, we are left with only the $z$ and $u$ integrals which have to be evaluated numerically.

Further simplifications occur in the interesting case of a homogeneous credit portfolio, in which all parameters for the obligors are the same, face value $F_{k}=F_{0}$, variance $\rho_{k}^{2}=\rho_{0}^{2}$, drift $\mu_{k}=\mu_{0}$ and initial value $V_{k 0}=V_{0}$. Of course, this does not mean that the actual paths explored by the stochastic processes are the same. The fractions are now all equal, $f_{k}=1 / K$, the same is true for the moments $m_{j k}(z, u)=m_{j 0}(z, u)$. Importantly, this allows us to calculate the strict limit $K \rightarrow \infty$ of the averaged loss distribution (11). The Gaussian in Eq. (15) becomes 


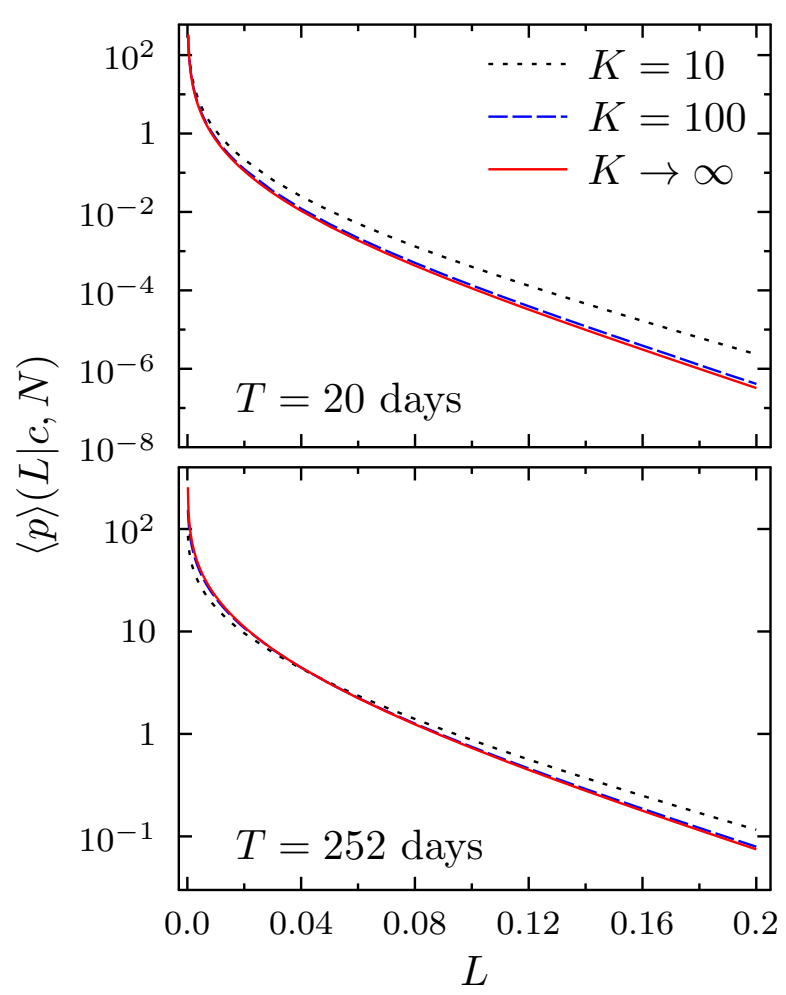

FIG. 4: The average loss distribution for $N=4.2$ and a correlation level of $c=0.26$ with drift $\mu_{0}=0.013 \mathrm{month}^{-1}$, volatility $\rho_{0}=0.1$ month $^{-1 / 2}, T=20$ trading days (top) and for $N=6$, a correlation level of $c=0.28$ with drift $\mu_{0}=0.17$ year $^{-1}$, volatility $\rho_{0}=0.35$ year $^{-1 / 2}$ and $T=1$ year (bottom). The dotted and dashed line are for $K=10$ and $K=100$, while the solid line shows the limit $K \rightarrow \infty$.

a $\delta$ function such that

$$
\begin{aligned}
\lim _{K \rightarrow \infty}\langle p\rangle(L \mid c, N)= & \frac{1}{2^{N / 2} \Gamma(N / 2)} \sqrt{\frac{N}{2 \pi}} \int_{0}^{\infty} d z z^{N / 2-1} e^{-z / 2} \\
& \times \int_{-\infty}^{+\infty} d u \exp \left(-\frac{N}{2} u^{2}\right) \delta\left(L-m_{10}(z, u)\right) \\
& =\frac{1}{2^{N / 2} \Gamma(N / 2)} \sqrt{\frac{N}{2 \pi}} \int_{0}^{\infty} d z z^{N / 2-1} e^{-z / 2} \\
& \times \exp \left(-\frac{N}{2} u_{0}^{2}\right) \frac{1}{\left|\partial m_{10}(z, u) / \partial u\right|_{z, u_{0}}}
\end{aligned}
$$

where $u_{0}$ is the zero of the first moment, $m_{10}\left(z, u_{0}\right)=0$.
This partly implicit formula yields a strict lower bound for the tail of the averaged loss distribution.

\section{RESULTS}

In Fig. 4 we show our result (15) for homogeneous credit portfolios of different sizes $K=10,100$, compared with the limiting curve 18 for a maturity time of $T=1$ month (top) and $T=1$ year (bottom). The parameters are chosen according to the data analysis, so we have $N=4.2$ and $N=6.0$. From the data set we find a drift of $\mu_{0}=0.013$ month $^{-1}$ and $\mu_{0}=0.17$ year $^{-1}$, volatility of $\rho_{0}=0.1$ month $^{-1 / 2}$ and $\rho_{0}=0.35$ year $^{-1 / 2}$ and an average correlation level of $c=0.26$ and $c=0.28$ depending on the time horizon. The face value is $F_{0}=75$ and the initial value is $V_{0}=100$. Both have the dimension currency. Notice that for the maturity time of one month the probability of a default is much smaller due to the lower volatility and the reduced time horizon. One clearly sees that the tails of the averaged loss distribution for finite $K$ very quickly reach the limiting curve for $K \rightarrow \infty$. We thus arrive at the truly disturbing observation that diversification generically cannot work for any realistic choice of correlation structure.

\section{CONCLUSIONS}

We uncovered and derived generic features of the loss distribution for credit portfolios. Our starting point was the Merton model which is known to give a realistic description. By transferring the concept of ensemble averaging to this problem, we were able to derive an averaged portfolio loss distribution which depends on only two parameters that fully account for the non-stationary dynamics of the markets. Data analysis strongly corroborates our approach. As an important application, we showed that diversification is bound to fail for a homogeneous portfolio. This is due to the correlations which are always present in reality. Pictorially speaking, they glue together the obligors and thereby let them act to some extent like just one obligor. Thus, we have no reason to hope that diversification can work for any other nonhomogeneous but realistic credit portfolio. All this is tantamount to saying that there is an intrinsic instability in the markets which cannot be overcome. We emphasize that we did not study "credit contagion" or avalanche effects after the onset of a crisis, as for example in Ref. [25]. We uncovered the substantial, unavoidable stability risk which is always there, even in periods in which the markets appear quiet. It thus seems reasonable to advertize a considerable enlargement of the equity held by the banks and other creditors.
[1] Michel Crouhy, Dan Galai, and Robert Mark. A comparative analysis of current credit risk models. Journal of Banking \& Finance, 24(1-2):59-117, January 2000. [2] John C. Hull. The credit crunch of 2007: What went 
wrong? Why? What lessons can be learned? The Journal of Credit Risk, 5(2):3-18, 2009.

[3] Barry Eichengreen, Ashoka Mody, Milan Nedeljkovic, and Lucio Sarno. How the Subprime Crisis went global: Evidence from bank credit default swap spreads. Journal of International Money and Finance, 31(5):1299-1318, September 2012.

[4] T. R. Bielecki and M. Rutkowski. Credit Risk: Modeling, Valuation and Hedging. Springer, 2004.

[5] C. Bluhm, L. Overbeck, and C. Wagner. An Introduction to Credit Risk Modeling. Chapman \& Hall/CRC, 2003.

[6] Darrell Duffie and Kenneth J. Singleton. Modeling Term Structures of Defaultable Bonds. Review of Financial studies, 12(4):687-720, 1999.

[7] D. Lando. Credit Risk Modeling: Theory and Applications. Princeton University Press, 2008.

[8] A. J. McNeil, R. Frey, and P. Embrechts. Quantitative Risk Management: Concepts, Techniques, and Tools. Princeton University Press, 2005.

[9] Amar Bhidé. How Banking Diversification Steered Us Wrong. Bloomberg Businessweek Magazine, 2009.

[10] Paul Glasserman. Tail approximations for portfolio credit risk. The Journal of Derivatives, 12(2):24-42, 2004.

[11] Philipp J. Schönbucher. Credit Derivatives Pricing Models: Models, Pricing and Implementation. The Wiley Finance Series. Wiley, 2003.

[12] Erik Heitfield, Steve Burton, and Souphala Chomsisengphet. Systematic and idiosyncratic risk in syndicated loan portfolios. Journal of Credit Risk, 2(3):3-31, 2006.

[13] Paul Glasserman and Jesus Ruiz-Mata. Computing the credit loss distribution in the Gaussian copula model: a comparison of methods. Journal of Credit Risk, 2(4), 2006.

[14] Robert C. Merton. On the pricing of corporate debt: the risk structure of interest rates. J. Finance, 29(2):449-470, 1974.
[15] Robert A. Jarrow, D. Lando, and Stuart M. Turnbull. A Markov Model for the Term Structure of Credit Risk Spreads. Review of Financial Studies, 10(2):481-523, 1997.

[16] Rudi Schäfer, Markus Sjölin, Andreas Sundin, Michal Wolanski, and Thomas Guhr. Credit risk - A structural model with jumps and correlations. Physica A, 383(2):533-569, September 2007.

[17] Alexander F. R. Koivusalo and Rudi Schäfer. Calibration of structural and reduced-form recovery models. Journal of Credit Risk, 8(4):31-51, 2012.

[18] Philipp J. Schönbucher. Factor Models: Portfolio Credit Risks When Defaults are Correlated. Journal of Risk Finance, The, 3(1):45-66, 2001.

[19] Desislava Chetalova, Thilo A. Schmitt, Rudi Schäfer, and Thomas Guhr. Portfolio return distributions: Sample statistics with non-stationary correlations. Arxiv preprint arXiv:1308.3961, 2013.

[20] Thilo A. Schmitt, Desislava Chetalova, Rudi Schäfer, and Thomas Guhr. Non-Stationarity in Financial Time Series and Generic Features. Arxiv preprint arXiv:1304.5130, 2013.

[21] J Wishart. The Generalised Product Moment Distribution in Samples from a Normal Multivariate Population. Biometrika, 20A(1/2):32-52, 1928.

[22] Standard \& Poor's 500 data from Yahoo! Finance, 2013.

[23] Michael C. Münnix, Rudi Schäfer, and Thomas Guhr. A Random Matrix Approach on Credit Risk. Arxiv preprint arXiv:1102.3900, 2011.

[24] Kiyosi Itô. Stochastic integral. Proceedings of the Imperial Academy, 20(8):519-524, 1944.

[25] Reimer Kühn and Peter Neu. Functional correlation approach to operational risk in banking organizations. Physica A, 322:650-666, May 2003. 